\title{
Topological Anderson Insulator in Three Dimensions
}

\author{
H.-M. Guo, ${ }^{1,2}$ G. Rosenberg, ${ }^{1}$ G. Refael, ${ }^{3}$ and M. Franz ${ }^{1}$ \\ ${ }^{1}$ Department of Physics and Astronomy, University of British Columbia, Vancouver, BC, Canada V6T 1Z1 \\ ${ }^{2}$ Department of Physics, Capital Normal University, Beijing 100048, China \\ ${ }^{3}$ Department of Physics, California Institute of Technology, Pasadena, California 91125, USA
}

(Received 1 August 2010; published 19 November 2010)

\begin{abstract}
We show that disorder, when sufficiently strong, can transform an ordinary metal with strong spin-orbit coupling into a strong topological "Anderson" insulator, a new topological phase of quantum matter in three dimensions characterized by disordered insulating bulk and topologically protected conducting surface states.
\end{abstract}

DOI: 10.1103/PhysRevLett.105.216601

PACS numbers: $72.80 . \mathrm{Sk}, 73.20 . \mathrm{Fz}$

Disorder is well known to play a fundamental role in low-dimensional electronic systems, leading to electron localization and consequent insulating behavior in the time-reversal invariant systems [1]. Disorder also underlies much of the phenomenology of the integer quantum Hall effect [2]. Recently, in a remarkable development, it has been noted first by numerical simulations [3] and shortly thereafter by analytical studies [4], that a phase similar to the two dimensional topological insulator [5,6] (also known as the quantum spin-Hall insulator $[7,8]$ ) can be brought about by introducing nonmagnetic disorder into a 2D metal with strong spin-orbit coupling. This new 2D topological phase, referred to as topological Anderson insulator (TAI), has a disordered insulating bulk with topologically protected gapless edge states that give rise to precisely quantized conductance $e^{2} / h$ per edge. In TAI, remarkably, conductance quantization owes its very existence to disorder.

A question naturally arises whether such behavior can occur in three spatial dimensions. More precisely, one may inquire whether an inherently 3D topological phase analogous to the strong topological insulator [9-11] (STI) could be reached by disordering a clean system that is initially in a topologically trivial phase. Below, we show the answer to the above question to be affirmative. Employing a combination of analytical and numerical methods we construct an explicit example of a disorder-induced topological phase in three dimensions with physical properties analogous to those of the strong topological insulator. We propose to call this new phase a "strong topological Anderson insulator" (STAI). We argue that some of the topologically trivial compounds with strong spin-orbit coupling discussed in the recent literature, such as, e.g., $\mathrm{Sb}_{2} \mathrm{Se}_{3}$, could become a STAI upon introducing disorder. In other compounds that already are STIs in their clean form, disorder can reinforce this behavior by rendering the bulk truly insulating. We note that the existence of a 3D disorder-induced topological phase has been previously conjectured in Ref. [4].

To study the emergence of the STAI we consider a variant of a model describing itinerant electrons with spin-orbit coupling on a cubic lattice discussed extensively in the recent literature [12-14]. It has four electron states per lattice site $\mathbf{r}_{j}$, compactly denoted as $\Psi_{j}=\left(\psi_{1 j}, \psi_{2 j}, \psi_{3 j}, \psi_{4 j}\right)^{T}$, and a Hamiltonian $H_{0}=$ $\sum_{\mathbf{k}} \Psi_{\mathbf{k}}^{\dagger} \mathcal{H}_{\mathbf{k}} \Psi_{\mathbf{k}}$ with $\Psi_{\mathbf{k}}$ the Fourier transform of $\Psi_{j}$,

$$
\mathcal{H}_{\mathbf{k}}=\sum_{\mu=0}^{3} d_{\mu}(\mathbf{k}) \Gamma_{\mu}+d_{4}(\mathbf{k}) \mathbb{1},
$$

and $d_{0}(\mathbf{k})=\epsilon-2 t \sum_{i} \cos k_{i}, d_{i}(\mathbf{k})=-2 \lambda \sin k_{i}(i=1,2$, $3)$ and $d_{4}(\mathbf{k})=2 \gamma \sum_{i}\left(1-\cos k_{i}\right)$. Here $\Gamma_{\mu}$ are $4 \times 4$ Dirac matrices in combined orbital and spin space, satisfying the canonical anticommutation relation $\left\{\Gamma_{\mu}, \Gamma_{\nu}\right\}=2 \delta_{\mu \nu}$. The system defined by $H_{0}$ is invariant under time reversal and spatial inversion. In the following we take two alternate points of view regarding Hamiltonian (1): (i) we view it as a simple toy model conveniently describing both topological and ordinary phases of noninteracting electrons in 3D and, (ii) we regard it as a lattice regularization of the effective low-energy Hamiltonian describing the physics of insulators in the $\mathrm{Bi}_{2} \mathrm{Se}_{3}$ family [15-17]. In the latter interpretation $\Psi_{j}$ labels the manifold of four active orbitals $\left(\left|P 1_{z}^{+}, \uparrow\right\rangle,\left|P 2_{z}^{-}, \uparrow\right\rangle,\left|P 1_{z}^{+}, \downarrow\right\rangle,\left|P 2_{z}^{-}, \downarrow\right\rangle\right)$.

The spectrum of $H_{0}$ has two doubly degenerate bands,

$$
E_{\mathbf{k}}=d_{4}(\mathbf{k}) \pm \sqrt{\sum_{\mu} d_{\mu}^{2}(\mathbf{k})} .
$$

At half filling, depending on the values of the parameters $\epsilon$, $t, \lambda$, and $\gamma$ the system can be a metal, a trivial insulator, as well as a strong and weak topological insulator $[12,14]$. Below, we focus on the topological phase transition between the ordinary insulator characterized by the $Z_{2}$ invariant $[9,10](0 ; 000)$ and the $(1 ; 000)$ STI phase. In the clean system modeled by the Hamiltonian (1) this transition occurs due to the band inversion at the $\boldsymbol{\Gamma}$ point of the Brillouin zone and can be driven by tuning parameter $\epsilon$ through a critical value $\epsilon_{c}=6 t$. We take $t, \lambda$, and $\gamma$ positive here and in what follows. We note that it is exactly this physics that underlies the STI behavior in the $\mathrm{Bi}_{2} \mathrm{Se}_{3}$ 
family of materials [15-17]. Our main result is the finding that a similar transition can be effected by introducing nonmagnetic disorder into a system that is topologically trivial in its clean form.

To simulate the effects of disorder we consider a Hamiltonian of the form

$$
H=H_{0}+\sum_{j} U_{j} \Psi_{j}^{\dagger} \Psi_{j},
$$

where $U_{j}$ is a random on-site potential uniformly distributed in the range $\left(-U_{0} / 2, U_{0} / 2\right)$. Following the discussion in Ref. [4] we start by treating the disorder within the self-consistent Born approximation (SCBA). The disorderaveraged electron propagator $g(\omega, \mathbf{k})=\left(\omega+i \delta+E_{F}-\right.$ $\left.\mathcal{H}_{\mathbf{k}}-\Sigma\right)^{-1}$ is then given in terms of disorder self-energy $\Sigma$, subject to the self-consistent equation

$$
\Sigma=\frac{U_{0}^{2}}{12} \sum_{\mathbf{k} \in \mathrm{BZ}}\left(E_{F}+i \delta-\mathcal{H}_{\mathbf{k}}-\Sigma\right)^{-1} .
$$

Here $E_{F}$ refers to the Fermi energy and $\delta=0^{+}$. The factor of 12 arises from the variance $\left\langle U^{2}\right\rangle=U_{0}^{2} / 12$.

For pointlike disorder symmetry consideration restricts the form of the self-energy to $\Sigma=\Gamma_{0} \Sigma_{0}+\mathbb{1} \Sigma_{4}$. The two nonvanishing components of the self-energy, $\Sigma_{0}$ and $\Sigma_{4}$, can be viewed as disorder-induced renormalizations of the "topological mass" $m \equiv d_{0}(\mathbf{k}=0)=\epsilon-6 t$ and the Fermi energy $E_{F}$, respectively. Within the SCBA the disorderaveraged system is described by the same propagator $g_{0}(\omega, \mathbf{k})=\left(\omega+i \delta+E_{F}-\mathcal{H}_{\mathbf{k}}\right)^{-1}$ as the clean system but with parameters $m$ and $E_{F}$ replaced as $m \rightarrow \bar{m}=m+$ $\Sigma_{0}$ and $E_{F} \rightarrow \bar{E}_{F}=E_{F}-\Sigma_{4}$. We note that $\Sigma_{0}$ and $\Sigma_{4}$ are generally complex-valued, the imaginary part reflecting the quasiparticle lifetime broadening due to disorder.

These considerations underlie the physical picture behind the emergence of the STAI. Starting from a clean ordinary insulator (characterized by $m>0$ ) disorder can induce a band inversion by driving the real part of the renormalized mass $\bar{m}$ negative. According to the standard classification of topological insulators [18] a band inversion at an odd number of time-reversal invariant momenta changes the $Z_{2}$ class of the material. If the renormalized Fermi energy $\bar{E}_{F}$ lies within the gap the resulting effective medium is a STI.

Equation (4) leads to the self-consistent equations for $\bar{m}$ and $\bar{E}_{F}$

$$
\begin{gathered}
\bar{m}=m-\frac{U_{0}^{2}}{12} \sum_{\mathbf{k}} \frac{\bar{m}+t c_{\mathbf{k}}}{D_{\mathbf{k}}}, \\
\bar{E}_{F}=E_{F}+\frac{U_{0}^{2}}{12} \sum_{\mathbf{k}} \frac{\bar{E}_{F}-\gamma c_{\mathbf{k}}}{D_{\mathbf{k}}},
\end{gathered}
$$

with $D_{\mathbf{k}}=\lambda^{2} s_{\mathbf{k}}^{2}+\left(\bar{m}+t c_{\mathbf{k}}\right)^{2}-\left(\bar{E}_{F}+i \delta-\gamma c_{\mathbf{k}}\right)^{2}$ and $s_{\mathbf{k}}^{2}=4 \sum_{i} \sin ^{2} k_{i}, \quad c_{\mathbf{k}}=2 \sum_{i}\left(1-\cos k_{i}\right)$. To gain some insight into the underlying physics it is useful to first study the approximate solution valid at weak-disorder (small $U_{0}$ ) obtained by replacing $\bar{m}$ and $\bar{E}_{F}$ on the right hand side by their respective bare values. One then obtains

$$
\bar{m} \simeq m-\frac{U_{0}^{2}}{24 \pi} \frac{t}{t^{2}-\gamma^{2}}, \quad \bar{E}_{F} \simeq E_{F}+\frac{U_{0}^{2}}{24 \pi} \frac{\gamma}{t^{2}-\gamma^{2}},
$$

where we kept only the leading ultraviolet-divergent terms after expanding the integrand around the $\boldsymbol{\Gamma}$ point. We observe that for $t>\gamma$ disorder indeed renormalizes the mass term downward; for $U_{0}>U_{c} \simeq\left[24 \pi m\left(t^{2}-\gamma^{2}\right) / t\right]^{1 / 2}$ band inversion occurs and one expects the system to become a STAI. This conclusion remains valid when Eqs. (5) and (6) are solved self-consistently.

We now describe our exact numerical diagonalization studies that establish the existence of disorder-induced topological phase in the model Hamiltonian (3). In a disordered system translational invariance is lost and the usual $k$-space methods $[9,10,18]$ for finding the topological invariants fail. An elegant method for calculating the $Z_{2}$ invariant of $2 \mathrm{D}$ topological insulators is given in Ref. [19], but as of now a 3D equivalent approach is unexplored. For this reason we employ a two-pronged strategy based on (i) generalizing the approach of Refs. $[3,4,20]$ to study the emergence of topologically protected extended gapless surface states that are the defining property of the strong topological insulator in 3D, and (ii) probing for the bulk Witten effect $[14,21]$ that arises from the unique "axion" electromagnetic response [12,22] characteristic of a STI.

To study the extended gapless surface states we employ the recursive Green's function method [23] and evaluate the conductance $G$ of a length- $L$ wire with a rectangular cross section $W_{x} \times W_{y}$ using the Landauer-Büttiker formalism [24,25]. For simplicity and concreteness, and to make contact with the previous works on $2 \mathrm{D}$ systems $[3,4,20]$, we use model parameters listed in the caption of Fig. 1, with values close to those describing $\mathrm{HgTe} / \mathrm{CdTe}$ quantum wells $[7,8]$.

Figure 1(a) shows conductance $G$ as a function of disorder strength $U_{0}$ in a wire with $W_{x}=4$ and $W_{y}=20$. For weak disorder the wire shows conductance characteristic of a disordered metal with significant fluctuations reflecting different realizations of the disorder potential $U_{i}$. Above $U_{0} \simeq 130 \mathrm{meV}$ the bulk conductance (measured with periodic boundary conditions along $x$ and $y$ ) drops to zero, indicating a disorder-induced insulating behavior in the bulk that persists up to $U_{0} \simeq 170 \mathrm{meV}$. If we change periodic boundary conditions to open along the $y$ direction a very different picture emerges. For $U_{0}$ in the range showing bulk insulating behavior the conductance is now pinned to the nonzero value $2 e^{2} / h$ with no observable fluctuations. We attribute this to the ballistic transport of topologically protected surface states. Figure 1(b) confirms that the current flows near the surface only. It furthermore shows that the current density is equally distributed among the two surfaces; each surface forms an independent conduction channel contributing one quantum $e^{2} / h$ to the total conductance $G$ of the sample. This is the disorder-induced topological phase introduced above. 

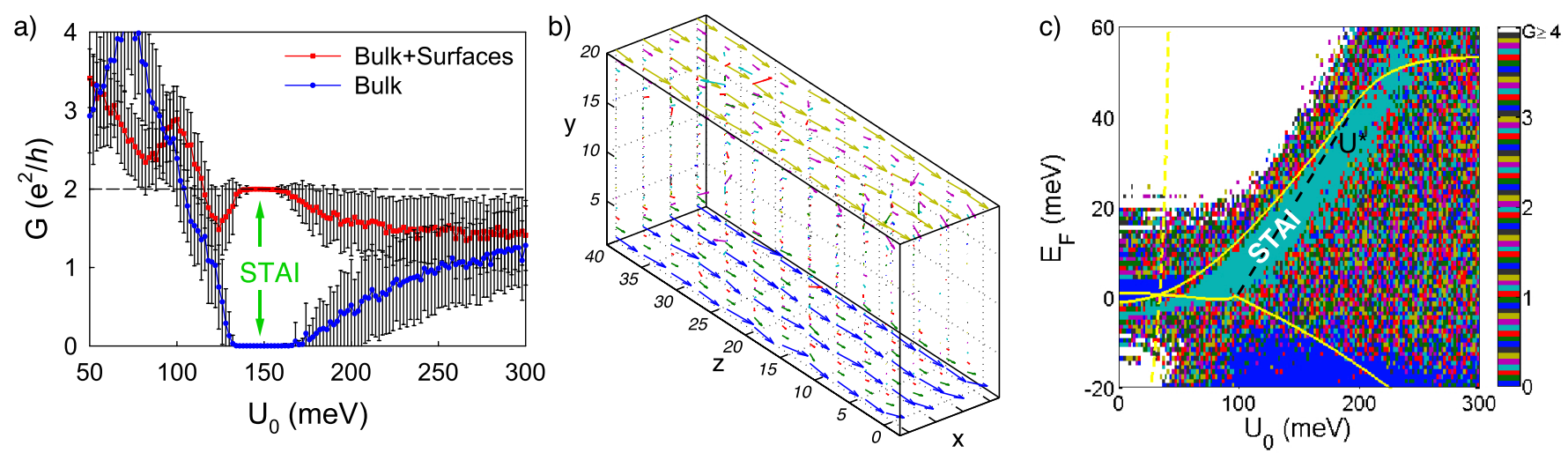

FIG. 1 (color online). (a) Conductance $G$ of a rectangular wire with dimensions $4 \times 20 \times 40$ as a function of disorder strength $U_{0}$ for periodic boundary conditions along $x$ and periodic (open) boundary conditions along $y$ as distinguished by blue (red) symbols, with $E_{F}=20 \mathrm{meV}$. Error bars reflect the conductance fluctuations in the ensemble of 100 independent disorder realizations. (b) Electrical current distribution in the STAI wire averaged over 100 independent disorder realization for $E_{F}=20 \mathrm{meV}$ and $U_{0}=150 \mathrm{meV}$. We use open boundary conditions along $y$ and periodic along $x$ direction. Arrows representing the local current density in different layers along the $y$ direction have been color coded for clarity. (c) False color plot of conductance as a function of disorder strength $U_{0}$ and the Fermi energy $E_{F}$. Each data point corresponds to a single realization of the disorder potential. The dashed line marks the band inversion boundary defined as $\operatorname{Re}(\bar{m})=0$ based on the SCBA. Solid lines represent the SCBA phase boundaries separating a band insulator and a metal defined by $\left|\operatorname{Re}\left(\bar{E}_{F}\right)\right|=|\operatorname{Re}(\bar{m})|$. In all three panels we use parameters $\epsilon=145 \mathrm{meV}, t=24 \mathrm{meV}, \lambda=20 \mathrm{meV}$, and $\gamma=16 \mathrm{meV}$, corresponding to $m=1 \mathrm{meV}$.

The odd number of 1D conductance channels per surface reflects the odd number of gapless states per surface of a STAI, confined to a 1D geometry imposed by the finite width of the wire in the $x$ direction. When we impose antiperiodic boundary conditions for the electron wave functions along the $x$ direction the conductance drops to zero in accord with the expectation for the surface state in a 3D topological phase. We checked that the conductance remains quantized at $2 e^{2} / h$ when we change the number of layers $W_{x}$ and there is no even or odd effect that one would expect from a collection of $2 \mathrm{D}$ topological insulators stacked along the $x$ direction $[3,4]$.

To further confirm the genuine 3D nature of the observed topological phase we probed for the Witten effect [21] in our model STAI. According to Refs. [12,22] the effective electromagnetic Lagrangian of a 3D strong topological insulator contains an unusual "axion" term $\sim \theta \mathbf{E} \cdot \mathbf{B}$ with $\theta=\pi$. According to Witten [21] a magnetic monopole inserted into a medium with nonzero $\theta$ binds electric charge $-e(\theta / 2 \pi+n)$ with $n$ integer. Using numerical methods described in Ref. [14] we measured the induced fractional charge in a configuration containing a monopole and an antimonopole depicted in Fig. 2(a). Our results presented in Figs. 2(b) and 2(c) clearly indicate fractional charge $\pm e / 2$ bound to the monopole, confirming the expected value of $\theta=\pi$. This result lends additional support to our identification of the STAI as a genuinely 3D topological phase characterized by the bulk axion term.

We now turn to the phase diagram of our model in the $\left(E_{F}, U_{0}\right)$ plane. In a real material these parameters can be tuned, at least in principle, by adjusting the chemical composition and disorder content. Because of the 3D nature of our system and the resulting large size of the Hamiltonian matrix that must be diagonalized, we were able to consider only a single realization of the disorder potential $U_{i}$ at each point of the $\left(E_{F}, U_{0}\right)$ phase diagram. Nevertheless this turns out to be sufficient for determining the location of the STAI phase to a very good accuracy. Our method relies on the fact that, as seen in Fig. 1(a), the conductance $G$ shows no observable fluctuations in the STAI phase but fluctuates significantly elsewhere. Figure 1(c) displays $G$ in a fashion that is designed to amplify the effect of fluctuations. The locus of the STAI phase is clearly visible as the region with $G=2 e^{2} / h$ and no discernible conductance fluctuations.

The weak-disorder boundary of the numerically determined STAI phase coincides with the metal-insulator transition deduced from the SCBA Eqs. (7). As in 2D, the weak-disorder STAI phase boundary marks the crossing of a band edge rather than a mobility edge [4]. The strongdisorder phase boundary is more interesting. According to SCBA states appear at the Fermi level beyond certain disorder strength $U^{*}\left(E_{F}\right)$ due to lifetime broadening. The band edge becomes ill defined here as the self-energy acquires a large imaginary part. Fig. 1(c) shows that the numerically determined STAI phase extends well beyond $U^{*}\left(E_{F}\right)$. The bulk electron states near the Fermi level do not contribute to conduction in this regime and must therefore be localized. We thus identify this region as the "true" topological Anderson insulator where electron localization plays the key role.

Although discussed here in the framework of a concrete model we expect the emergence of disorder-induced topological phases to be quite generic in three spatial dimensions. We have verified by an explicit calculation that including uniaxial anisotropy characteristic of the materials in the $\mathrm{Bi}_{2} \mathrm{Se}_{3}$ family [15-17] leads to qualitatively similar behavior to that displayed in Fig. 1. This 
a)

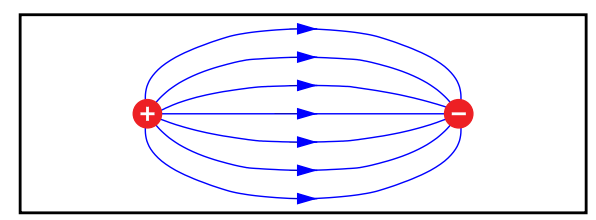

b)
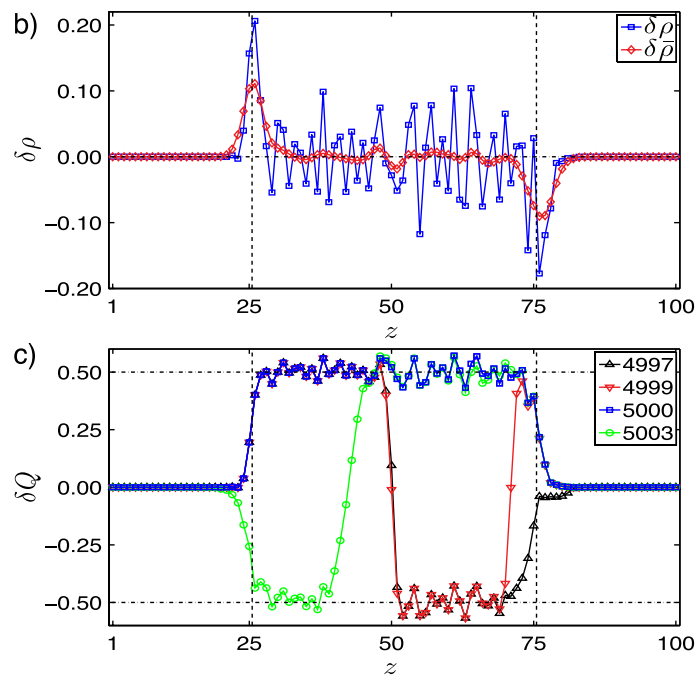

FIG. 2 (color online). Witten effect in the strong topological Anderson insulator. (a) A prism-shaped sample with monopole $(+)$, antimonopole $(-)$, and field lines indicated. The asymmetric field distribution allows us to use periodic boundary conditions in all directions and thus avoid difficulties associated with the surface states. (b) Electric charge density $\delta \rho(z)=$ $\sum_{x, y}\left[\rho(\mathbf{r})-\rho_{0}(\mathbf{r})\right]$ induced by the monopole-antimonopole pair in a $5 \times 5 \times 100$ sample at half-filling. Here $\rho$ and $\rho_{0}$ represent the charge density with and without the pair, respectively. A smoothed charge density function $\delta \bar{\rho}(z)$, obtained by convolving $\delta \rho(z)$ with a Gaussian of width $\sigma=1.5$, is also plotted to emphasize charge bound to the monopole. (c) Integrated charge $\delta Q(z)=\sum_{z^{\prime} \leq z} \delta \rho\left(z^{\prime}\right)$ in units of $e$ for $E_{F}$ in the range $19-25 \mathrm{meV}$, corresponding to the STAI phase. The number of filled electron states is indicated in the legend (5000 represents the half-filling). The steps of magnitude $\pm e / 2$ at the monopole-antimonopole locations show the expected localized fractional charge due to the Witten effect. In both panels we use $U_{0}=150 \mathrm{meV}$ and other parameters as in Fig. 1.

suggests that clean $\mathrm{Sb}_{2} \mathrm{Se}_{3}$, predicted to be a trivial insulator [15] (but nevertheless close to the topological phase), could become a STAI upon introducing nonmagnetic disorder. Whether or not disorder can effect a band inversion in $\mathrm{Sb}_{2} \mathrm{Se}_{3}$ will depend crucially on the magnitude of its native band gap. Our simulations suggest that when the band gap size becomes larger than $\sim 20 \mathrm{meV}$ the amount of disorder required to produce a band inversion is so large that the electron states become localized before the STAI phase can be reached. From this point of view the best prospects for experimental realization of STAI physics lie with materials that are very small or zero band gap semiconductors with strong spin-orbit coupling. Although bulk
$\mathrm{HgTe}$ as well as the recently discussed Heusler compounds $[26,27]$ exhibit this type of behavior their low-energy physics is not well described by our four-band model and further theoretical work is needed to determine whether disorder could drive the transition into the topological phase.

The authors acknowledge illuminating discussions with I. Garate, A. Kitaev, J. E. Moore, A. Vishwanath, C. Weeks, and S.-C. Zhang. The work was supported in part by NSERC, CIfAR (M.F.), China Scholarship Council (H.M.G.), the Packard Foundation, and the Research Corporation (G. R.).

[1] P. A. Lee and T. V. Ramakrishnan, Rev. Mod. Phys. 57, 287 (1985).

[2] F. Evers and A. D. Mirlin, Rev. Mod. Phys. 80, 1355 (2008).

[3] J. Li, R.-L. Chu, J. K. Jain, and S.-Q. Shen, Phys. Rev. Lett. 102, 136806 (2009).

[4] C. W. Groth et al., Phys. Rev. Lett. 103, 196805 (2009).

[5] J. E. Moore, Nature (London) 464, 194 (2010).

[6] M.Z. Hasan and C. L. Kane, Rev. Mod. Phys. 82, 3045 (2010).

[7] B. A. Bernevig, T. L. Hughes, and S.-C. Zhang, Science 314, 1757 (2006).

[8] M. König et al., Science 318, 766 (2007).

[9] L. Fu, C. L. Kane, and E. J. Mele, Phys. Rev. Lett. 98, 106803 (2007).

[10] J.E. Moore and L. Balents, Phys. Rev. B 75, 121306(R) (2007).

[11] R. Roy, Phys. Rev. B 79, 195322 (2009).

[12] X. L. Qi, T. L. Hughes, and S.-C. Zhang, Phys. Rev. B 78, 195424 (2008).

[13] P. Hosur, S. Ryu, and A. Vishwanath, Phys. Rev. B 81, 045120 (2010).

[14] G. Rosenberg and M. Franz, Phys. Rev. B 82, 035105 (2010).

[15] H. Zhang, C.-X. Liu, X.-L. Qi, X. Dai, Z. Fang, and S.-C. Zhang, Nature Phys. 5, 438 (2009).

[16] Y. Xia et al., Nature Phys. 5, 398 (2009).

[17] Y.L. Chen et al., Science 325, 178 (2009).

[18] L. Fu and C. L. Kane, Phys. Rev. B 76, 045302 (2007).

[19] T. A. Loring and M. B. Hastings, arXiv:1005.4883.

[20] H. Jiang, L. Wang, Q.-F. Sun, and X. C. Xie, Phys. Rev. B 80, 165316 (2009).

[21] E. Witten, Phys. Lett. B 86, 283 (1979).

[22] A. M. Essin, J.E. Moore, and D. Vanderbilt, Phys. Rev. Lett. 102, 146805 (2009).

[23] We use the variant of the method described by G. Metalidis and P. Bruno, Phys. Rev. B 72, 235304 (2005).

[24] R. Landauer, Philos. Mag. 21, 863 (1970).

[25] M. Büttiker, Phys. Rev. B 38, 9375 (1988).

[26] S. Chadov et al., Nature Mater. 9, 541 (2010).

[27] H. Lin et al., arXiv:1003.0155 [Nature Mat. (to be published)]. 